\title{
Localized Corrosion on 2219 Aluminium Alloy Coated with a Titanium Based Conversion Coating
}

\author{
$\underline{\text { R. Grillii }},{ }^{1, *}$ J. F. Watts, ${ }^{1}$ M. A. Baker, ${ }^{1}$ B. Dunn ${ }^{2}$ \\ ${ }^{1}$ The Surface Analysis Laboratory, University of Surrey, Guildford, Surrey, GU2 7XH, UK \\ ${ }^{2}$ European Space Agency, Noordwijk, The Netherlands \\ *Corresponding author (rossana.grilli@surrey.ac.uk)
}

High strength aluminium alloys are widely employed in aerospace applications. However specific environmental conditions may trigger corrosion and therefore these alloys require additional protection. Most current conversion coatings used for this purpose are $\mathrm{Cr}(\mathrm{VI})$ based, and thus environmentally undesirable. A possible replacement is a Ti based coating, Nabutan STI/310. The aim of this work is to gain a better understanding of corrosion mechanisms of the Al alloy surfaces coated with Nabutan, focusing on the role of intermetallic precipitates in pitting corrosion. The second phase precipitates in Al 2219 alloy were characterized by means of Auger electron spectroscopy (AES), scanning Auger microscopy (SAM) and energy dispersive x-ray spectroscopy (EDX). The surface was then coated and the same precipitate was analysed to observe how the coating had deposited on the intermetallics and the matrix. Subsequently the behaviour of the precipitate in a $3.5 \% \mathrm{NaCl}$ solution was investigated as a function of exposure time; 15 minutes, 45 minutes, 2, 4 and 8 hours. At the end of each exposure the sample was returned to microscope and the same precipitates re-analysed. The results showed how the coating deposited preferentially on the matrix. During the immersions, the coating itself suffered of a progressive degradation. Corrosion products rich in aluminium and oxygen were found to increasingly accumulate in a random manner over the matrix, as a function of time. Fe, and Mn from the alloy and Ti from the conversion coating did not show any major activity. $\mathrm{Cu}$ deposits were found after 8 hours of immersion.

Keywords: Al alloy, SAM, pitting, conversion coating.

\section{Introduction}

Aluminium alloys are widely employed within the spacecraft industry due to the possibility of improving mechanical and corrosion properties based upon the requirements of each particular application. More specifically the 2000 series (high copper content) and 7000 series (high zinc content) are used for structural components due to their high toughness and good fatigue strength. Generally these alloys offer a good corrosion resistance for most environmental exposures. However specific environmental conditions, such as long period storage in humid and salty environments, may trigger corrosion and therefore these alloys require additional protection.

Pitting corrosion is considered to be one of the principal mechanisms for damage of high strength alloys. Heterogeneities present at the surface of such Al alloys include precipitates and constituent particles (intermetallic particles formed by alloying and impurity elements, of 1-30 $\mu \mathrm{m}$ size). At these sites micro-flaws in the oxide film probably exist, and there are potential differences between the particles and the matrix. 
The micro-flaws and the galvanic couples are responsible not only of the nucleation but also of the growth of pits, when the alloy is exposed to an aggressive electrolyte [1].

The general reaction for the corrosion of aluminium is:

$$
2 \mathrm{Al}+3 \mathrm{H}_{2} \mathrm{O}+3 / 2 \mathrm{O}_{2} \rightarrow 2 \mathrm{Al}(\mathrm{OH})_{3}
$$

In the $2 \mathrm{XXX}$ series $\mathrm{Al}$ alloys the two major types of precipitates are $\theta^{\prime}\left(\mathrm{Al}_{2} \mathrm{Cu}\right)$ and $\mathrm{S}\left(\mathrm{Al}_{2} \mathrm{CuMg}\right)$. The $\theta^{\prime}$ is cathodic to the alloy matrix and causes corrosion of the $\mathrm{Al}$ alloy at the precipitate/alloy interface [1]. The $\mathrm{S}$ phase precipitates are particularly susceptible to pitting corrosion [3,4]. 2219 has an alloy composition of $\mathrm{Al}$ base, with $\mathrm{Cu}$ 5.8-6.8 wt.\%, Mn 0.2-0.4 wt.\%, Fe 0.0-0.3 wt.\% and trace concentrations of other elements. The major second phase precipitates in these alloys are $\mathrm{Al}-\mathrm{Cu}-\mathrm{Fe}-\mathrm{Mn}$, with a variable composition and size from 1 to $30 \mu \mathrm{m}$. Such Al-Cu-Fe-Mn second phase particles are generally accepted to be cathodic with respect to the matrix [3-5].

Numerous conversion coatings have been developed with the main purpose not only being the protection of aluminium from corrosion but also the improvement of the adhesion of paints and adhesives. Most conversion coatings used in the aerospace industry are chromium (VI) based. While these conversion coatings enhance the corrosion and adhesion properties of the alloy, the presence of $\operatorname{Cr}(\mathrm{VI})$ in the formulations, makes these potentially harmful to humans and environmentally undesirable [6,7]. Recent EU restrictions on the use of chromium (VI) established the legislative requirement to replace such coatings with a chromium-free conversion coating [8]. One of the possible candidate replacements is Nabutan STI/310, manufactured by NABU Oberflächentechnick $\mathrm{GmbH}$. This conversion coating is a mixture of organic and inorganic acids, including hexafluorotitanic acid.

This work represents part of an ongoing project to assess the best substitute for the chromium based treatments. The behaviour of the 2219 aluminium alloy surface after progressive exposure to a $3.5 \% \mathrm{NaCl}$ solution has been previously studied by means of SAM and EDX analysis. The current study was undertaken on the same alloy coated with Nabutan. The coated alloy was immersed in the $\mathrm{NaCl}$ solution for different time intervals, the same intermetallics were relocated and analysis repeated after each exposure. This procedure has been developed in order to monitor changes in shape and composition of the precipitates caused by corrosion. The behaviour of three $\mathrm{Al}-\mathrm{Cu}-\mathrm{Fe}-$ Mn intermetallics was followed. Since, in each case, the results were very similar, only the results for one intermetallic feature are presented.

\section{Experimental}

A $1 \mathrm{~cm}^{2}$ specimen of the 2219 aluminium alloy was wet ground with 600, 1200, 2500 and 4000 grit silicon carbide papers, then polished with diamond paste to a $1 \mu \mathrm{m}$ finish. This was followed by ultrasonic cleaning in acetone and rinsing in ultra pure water. Sites of intermetallic particles were identified and marked using a Vickers microhardness tester. This allowed these regions to be readily located for subsequent 
analysis. The specimen was then analysed by means of scanning Auger microscopy and energy dispersive $\mathrm{x}$-ray spectroscopy, in the same microscope. The alloy was then coated with Nabutan and the analytical procedure was repeated.

The $3.5 \% \mathrm{NaCl}$ solution was prepared from analytical grade $\mathrm{NaCl}$ and ultra-pure water. The Al 2219 specimen was immersed in this solution for different periods of time and after each immersion it was washed in ultra-pure water, vertically drained onto blotting paper and then analyzed by means of AES, SAM and EDX.

Scanning Auger microscopy was performed using a Thermo Scientific MICROLAB 350 microscope fitted with an EDX detector (Thermo Fisher Scientific NORAN System Six). This enabled Auger electron and EDX spectra and images to be acquired from the same regions of the sample. An electron beam energy of $10 \mathrm{kV}$ was used for the acquisition of Auger data and $15 \mathrm{kV}$ for EDX spectra and images. The AES survey spectra $(30-1700 \mathrm{eV})$ were recorded with a retard ratio of $4(1 \mathrm{eV}$ channel width), whilst a retard ratio of 2.8 was used for SAM; the topographic effects were minimised by applying the well known $(\mathrm{P}-\mathrm{B}) /(\mathrm{P}+\mathrm{B})$ correction algorithm, where $\mathrm{P}$ is the Auger peak intensity and $\mathrm{B}$ is the background intensity. A Thermo Avantage datasystem was used for the acquisition and processing of SAM and AES data and Noran System Six was used for EDX analysis. Before the experiment the surface of the specimen was lightly sputtered with $1 \mathrm{kV}$ argon ions for 5-10 seconds, in order to reduce carbon contamination.

\section{Results}

Before exposure of the specimen to the $3.5 \% \mathrm{NaCl}$ solution an examination of the surface by SEM showed the presence of particles of various sizes, from 0.1 to $30 \mu \mathrm{m}$. A second phase particle was marked, in order to assess the development of the corrosion after exposure to $\mathrm{NaCl}$ solution. SEM, AES, SAM and EDX spectra and images were recorded from features of interest, and then the same set of analysis was repeated after the application of Nabutan on the surface and after the following immersion times: 15 minutes, 45 minutes, 2 hours, 4 hours and 8 hours. After 32 hours of immersion the marked particle could not be located, as a result of the substantial deposition of corrosion products.

Figure 1 shows an example of point analysis taken, before exposure, from the intermetallic and surrounding region. The central image shows a SEM micrograph of the particle (4000x magnification), its maximum length being $10 \mu \mathrm{m}$. Several points where chosen, on and around the particle and AES and EDX spectra were recorded from them, with a spot size of $155 \mathrm{~nm}$. Auger/EDX spectra from two points are shown in Figure 1, the spectra at the top (a-d) are from the as polished specimen, and the spectra at the bottom (e-h) are from the same points after coating with Nabutan. Figure 1 (a) and (g) are AES spectra representative of the matrix. Spectrum 1(a) exhibits large $\mathrm{Al} K L L$ and $\mathrm{O} K L L$ peaks and less intense $\mathrm{C} K L L$ and $\mathrm{Cu} L M M$ peaks. Spectrum $1(\mathrm{~g})$ shows large $\mathrm{C} K L L, \mathrm{O} K L L$ and $\mathrm{Al} K L L$ peaks, with lower intensity Ti $L M M$ and F 
KLL. Figure 1 (b) and (h) are the EDX surveys from the same point. They both show high intensity $\mathrm{Al} \mathrm{K} \alpha$ peaks and small $\mathrm{Cu} \mathrm{K} \alpha / \mathrm{L} \alpha$ peaks. The AES spectra from the intermetallic $1(\mathrm{~d})$ is dominated by a large $\mathrm{Cu} K L L$ peak, while spectrum 1(f) is similar to 1(g) (the coated matrix). The corresponding EDX spectra in Figure 1 (c) and (e) show strong $\mathrm{Al} \mathrm{K} \alpha$ and $\mathrm{Cu} \mathrm{K} \alpha$ peaks, indicating the thickness of the coating to be $<<$ $1 \mu \mathrm{m}$.

Figure 2 shows the high resolution Al $K L L$ spectra from the matrix in a polished 2(a) and coated 2(b) condition. The Al peak of the as polished alloy has two components: $1386 \mathrm{eV}$ (oxide) and $1394 \mathrm{eV}$ (metal). The spectrum from the coated specimen has a single peak at $1387 \mathrm{eV}$. The intermetallic doesn't show any aluminium for the as polished surface, and the Al spectrum for the coated specimen is similar to the one from the matrix.

Figure 3 displays the sequences (rows from top to bottom) of SEM micrographs and SAM maps for $\mathrm{Al} K L L, \mathrm{Cu} K L L, \mathrm{O} K L L, \mathrm{C} K L L$, Ti $K L L$, and the maps of $\mathrm{F} K L L$ and $\mathrm{Cl} K L L$ when these elements were detected. The columns from left to right are the SEM micrograph and SAM maps of the intermetallic before the application of the coating, after the coating with Nabutan, after immersion in a $3.5 \% \mathrm{NaCl}$ solution for 15 minutes, after a 45 minutes immersion, after 2 hours, after 4 hours and after 8 hours. The magnification of the SEM micrographs is 4000x for the first two and 5000x for the others. The SEM sequence shows the first corrosion deposits after 45 minutes of immersion in the corrosion medium. After 2 hours the surface of the matrix starts degrading progressively and after 8 hours it is extensively damaged. The aluminium, on the as polished surface, is located on the matrix. Once the surface is coated the $\mathrm{Al}$ distribution becomes irregular. After the immersions in the $\mathrm{NaCl}$ solution $\mathrm{Al}$ appears more intense on the deposits or on the damaged areas. Copper is sharply concentrated on the intermetallic. After the coating process the $\mathrm{Cu} K L L$ peak is no more detectable. It reappears weakly after 2 hours of immersion all over the surface and after 8 hours it is concentrated on part of the damaged areas. Oxygen is concentrated on the matrix of the just polished surface. After the coating its pattern is inverted, being more intense on the intermetallic. After the immersion steps it concentrates on the deposit areas, in the same way saw with the aluminium. Carbon is initially concentrated on the intermetallic, probably as a contamination. After the coating it is more intense on the matrix. With the first immersion the difference in intensity between the two areas intermetallic/matrix becomes weaker, and after 45 minutes of immersion carbon is uniformely spread across the surface, randomly covered by the corrosion deposits. Titanium makes its appearance after the coating process. Its peak is more intense on the matrix. After the first immersion it is no more defined in any pattern. After the coating process fluorine is detected, slightly more intense on the intermetallic, to disappear after the first immersion. After 8 hours in $\mathrm{NaCl}$ a weak chlorine signal is detected. This observation, maybe correlated to copper will be discussed later.

Figure 4a shows the SEM micrograph and EDX maps of the as polished surface. They display aluminium on the matrix and $\mathrm{Cu}, \mathrm{Fe}$ and $\mathrm{Mn}$ on the intermetallic. A low signal of oxygen is also detected on the intermetallic. Figure $4 \mathrm{~b}$ shows the EDX map 
sequence of oxygen through the stages of the experiment. It is evident that a consistent deposition of corrosion products occurs on the matrix in the 2 hour to 8 hour interval. Figure $4 \mathrm{c}$ shows the $\mathrm{Cl} \mathrm{K \alpha}$ map of the surface after 8 hours of immersion. Chlorine components now cover the precipitate.

\section{Discussion}

The chosen intermetallic was of an irregular shape, with a maximum length of $10 \mu \mathrm{m}$. The intermetallic components are aluminum, copper and a minor amount of iron and manganese. Oxide was also detected in the deep layers of the surface (up to $1 \mu \mathrm{m}$ ), probably from contamination as this element is evident in the EDX spectra. The top layers (1-10 $\mathrm{nm}$ depth) are made almost exclusively by copper. The surrounding matrix is mainly aluminium, with smaller amounts of copper and iron, and a thin surface layer of $\mathrm{Al}_{2} \mathrm{O}_{3}$. The passivation layer probably didn't have enough time to develop since the specimen was introduced in the vacuum system shortly after polishing. Smaller precipitates (about $1 \mu \mathrm{m}$ diameter) lay around the selected one; they are composed of iron and manganese.

The application of the Nabutan coating doesn't make any significant change in the deep layers of the alloy, indicating that the coating layer is less than $1 \mu \mathrm{m}$ thick. Significant variations are found on the top layers of the coating. Aluminium, is now distributed in a non-specific pattern, uniquely in the oxide state, as confirmed by the high resolution spectra of Figure 2b. Copper is no longer detectable by Auger analysis, oxygen is now more intense on the intermetallic rather than on the matrix and carbon, increased in intensity, is concentrated on the matrix. Two new components appear: titanium, more intense on the matrix, and fluorine, concentrated on the intermatallic.

Nabutan is a titanium based coating, the treatment contains exafluorotitanic acid and organic acids [3]. Usually a conversion coating for aluminium alloys contains HF, as it easily dissolves aluminum oxides. Its function is to etch the alumina surface layer, allowing the coating active component to react directly with the alloy. Nabutan STI/310 does not apparently contain HF. Having this set of informations as starting point, a mechanism for the deposition of the coating is proposed based on the data. Hexafluorotitanate is a bivalent anion, titanium is surrounded by six fluorine atoms in an octaedral structure. The compound is a metal complex and all the bonds are equivalent coordinate covalent bonds. When the complex approaches the alloy surface layer the six fluorine atoms, the ligands, tend to form the same complex with aluminium as central metal, giving a more stable complex. The released titanium will form an oxide layer on the alloy. The surface of the intermetallic is made of copper. The formation and precipitation of $\mathrm{CuF}_{2}$ does not occur since copper is not detected after the coating. The mechanism of reaction on a copper surface would lead to copper fluoride complexes, such as $\mathrm{CuF}_{3}^{-}, \mathrm{CuF}_{4}^{-}$and $\mathrm{CuF}_{6}^{-}$. If the first two are formed the titanium would react with the intermetallic surface with 2 or $3 \mathrm{~F}$ atoms still bound to it. This would explain the higher concentration of fluorine on the intermetallic. The titanium SAM map reflects the major affinity of the $\mathrm{TiF}_{6}{ }^{-}$for aluminium oxide. The second step of the mechanism is the deposition of an organic layer on top of the $\mathrm{TiO}_{2}$. The organic acid, probably a polymer, will be chemisorbed on the surface, to improve the adhesion of paints on the alloy. The chemisorption is higher on the matrix. At 
some point in the deposition process there is an erratic deposition of $\mathrm{Al}_{2} \mathrm{O}_{3}$, as shown on the SAM map of the coated specimen.

The progressive immersion of the alloy in a $3.5 \% \mathrm{NaCl}$ solution produces the increasing deposition of $\mathrm{Al}(\mathrm{OH})_{3}$. The deposits gather across the matrix surface rather than around the $\mathrm{Cu}$ intermetallics, as would be expected. Titanium is more cathodic than copper. One possibility is the formation of metallic $\mathrm{Ti}$ at the interface alloy/coating: the Nabutan bath is acidic ( $\mathrm{pH} 2.6-3)$, and in acid environment the ion $\mathrm{TiO}^{2+}$ could reduce to $\mathrm{Ti}(\mathrm{s})$ and water. The corresponding oxidation could be $\mathrm{Al}(\mathrm{s})$ to $\mathrm{Al}_{2} \mathrm{O}_{3}$, and this would explain the erratic distribution of the oxide after the coating. Iron and manganese don't show any activity, and these elements are never detected by AES analysis and the maps of $\mathrm{Fe} \mathrm{K} \alpha$ and $\mathrm{Mn} \mathrm{K} \alpha$ do not show any variation for the different immersion steps. Copper has disappeared from the AES spectra after coating, but it appears again after 2 hours of immersion. After 8 hours backdeposited $\mathrm{Cu}$ clusters are more evident, they probably come from the gradual dissolution of the alloy. Chlorine is also detected with the EDX analysis on the intermetallic. This indicates that the coating damage allows the mobile chlorine ion to reach the intermetallic and precipitate as insoluble cuprous chloride [9]. The C KLL maps show how the organic film degrades progressively with immersion time. The $\mathrm{O} K \alpha$ map sequence highlights how the oxygen concentration rises around a virtual rectangle containing the intermetallic. This rectangle corresponds to the area where the electron beam was focused during the first two steps of analysis. Probably there was some sort of interaction between the electron beam and the polymer layer which made it more stable in the corrosive medium, such as localized cross-linking.

\section{Conclusion}

- The $2219 \mathrm{Al}$ alloy shows the presence of large Al-Cu-Fe-Mn precipitates (5-30 $\mu \mathrm{m}$ in size) and smaller Al-Fe-Mn precipitates (about $1 \mu \mathrm{m}$ ). The surface was analysed by means of SEM, SAM and EDX after every step of the experiment.

- The surface was coated with Nabutan. The coating preferentially deposited on the matrix rather than on the intermetallic. Deposition of $\mathrm{Al}_{2} \mathrm{O}_{3}$ is also recorded.

- Immersion of the coated specimen in a $3.5 \% \mathrm{NaCl}$ solution produces deposition of $\mathrm{Al}(\mathrm{OH})_{3}$ deposits on the matrix and backdeposition of copper after 8 hours of immersion.

\section{References}

[1] B.Kuźnicka Materials Characterization (2009) available online, article in press

[2] C. Vargel, Corrosion of Aluminium (2004) Elsevier

[3] M.Shao, Y.Fu, R.Hu, C.Lin Materials Science and Engineering A344 (2003) 323

[4] D.Zhu, W.J. van Ooij Corrosion Science (2003) 45; 2163

[5] C.-M.Liao, R.P.Wei Electrochimica Acta (1999) 45; 881

[6] A. Rabbetts, Trans Inst Met Finish (1999) 76; B4

[7] Official Journal of the European Union, L37/19 from 13-02-2003

[8] European Space Agency, "Space product Assurance”, ECSS-Q-70-71A rev. 1, 18 June (2004)

[9] J. E. Castle, Surface and Interface Analysis (1986) 9; 345 


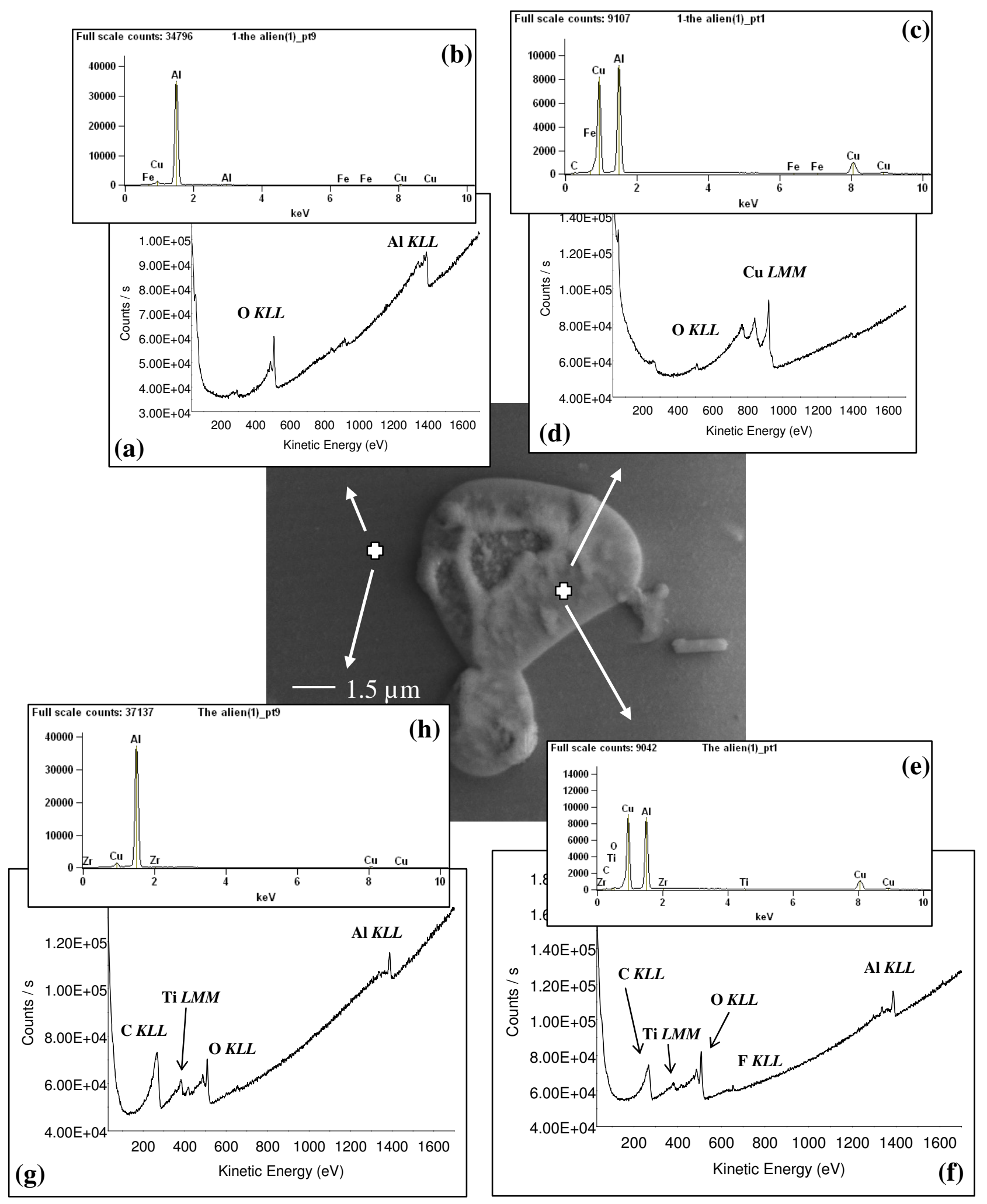

Figure 1: A SEM image and AES/EDX point analysis from the area around a recently polished Al-CuFe-Mn intermetallic second phase particle in the $2219 \mathrm{Al}$ alloy, The top spectra (a-b-c-d) are from the just polished surface; the bottom spectra (e-f-g-h) are from the same points after coating with Nabutan. 


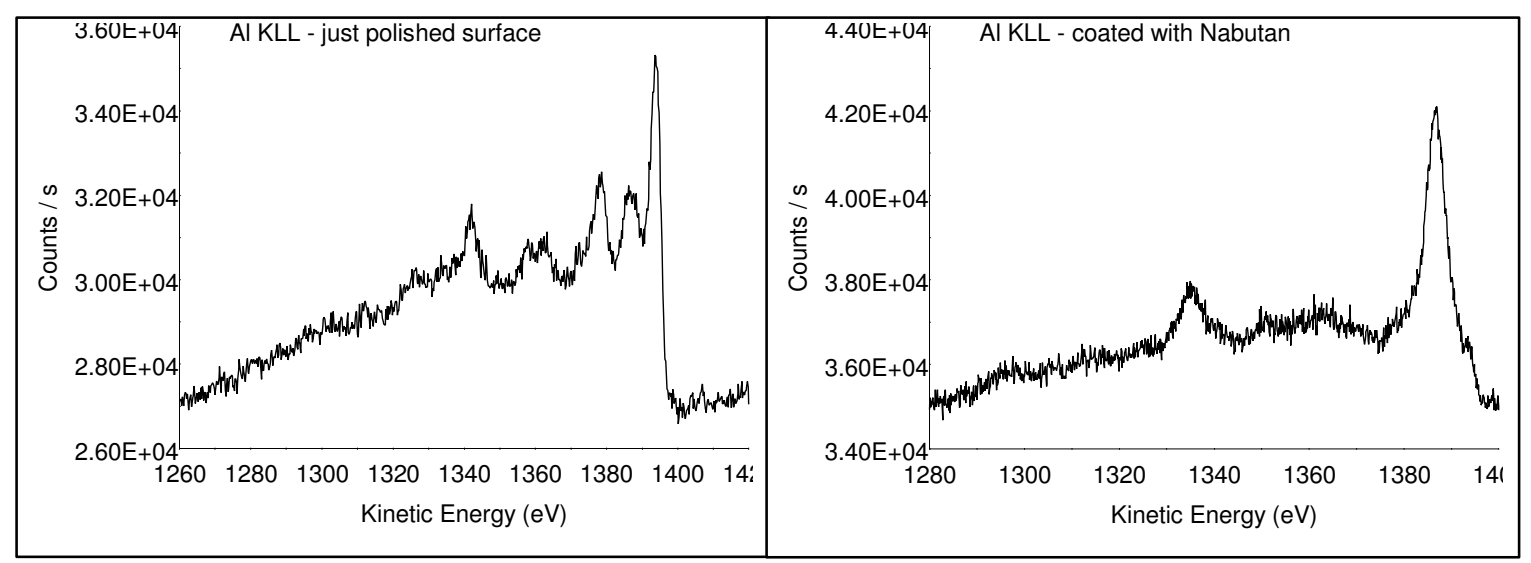

Figura 2Auger high resolution scans of Al KLL peak from a point on the matrix of the just polished surface (left) and of the coated surface (right). 


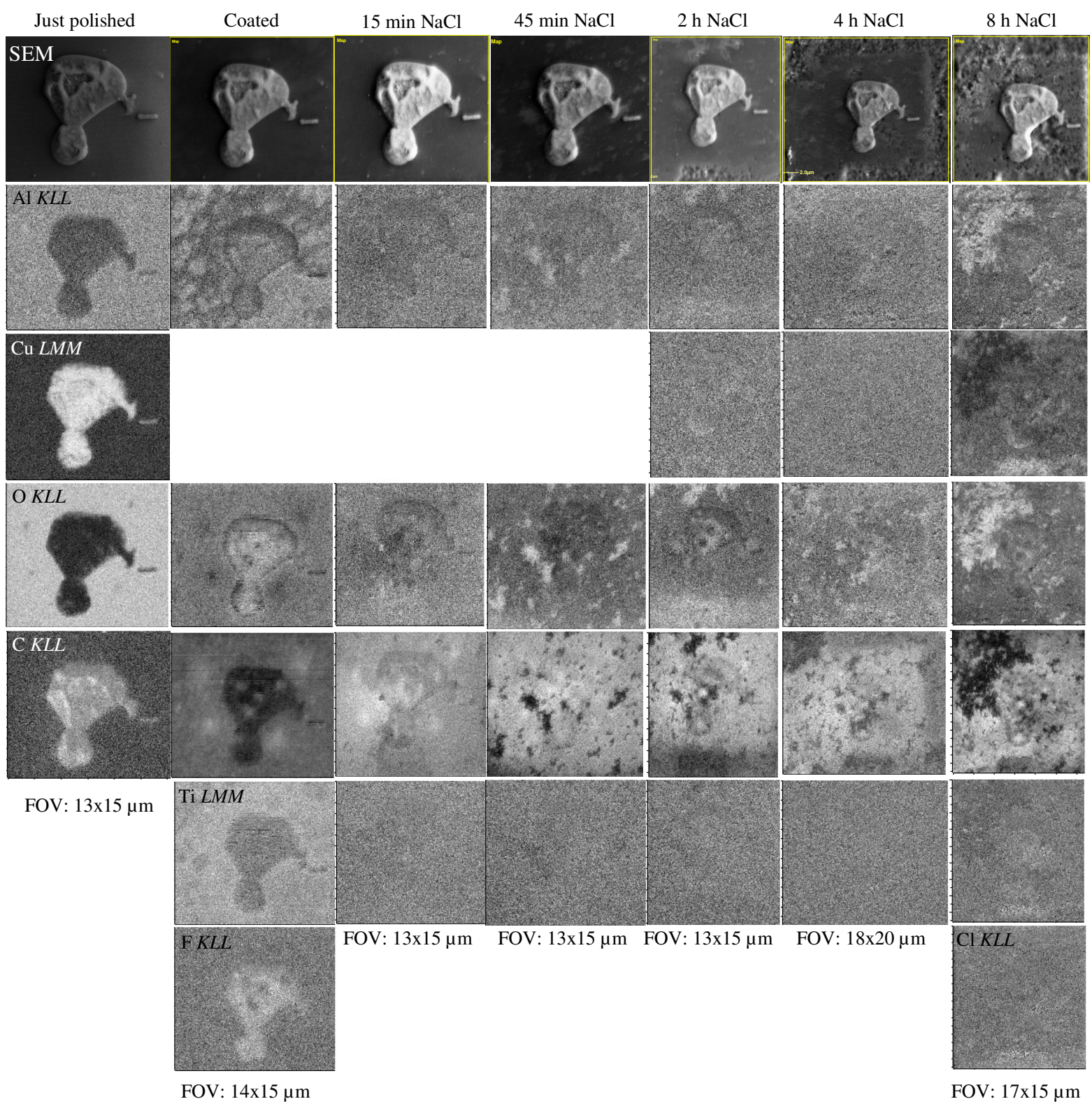

Figura 3: SEM micrographs (first row) and SAM maps of $\mathrm{Al}, \mathrm{Cu}, \mathrm{O}, \mathrm{C}, \mathrm{Ti}, \mathrm{F}$ and $\mathrm{Cl}$ at different times of immersion in the $3.5 \% \mathrm{NaCl}$ solution. 

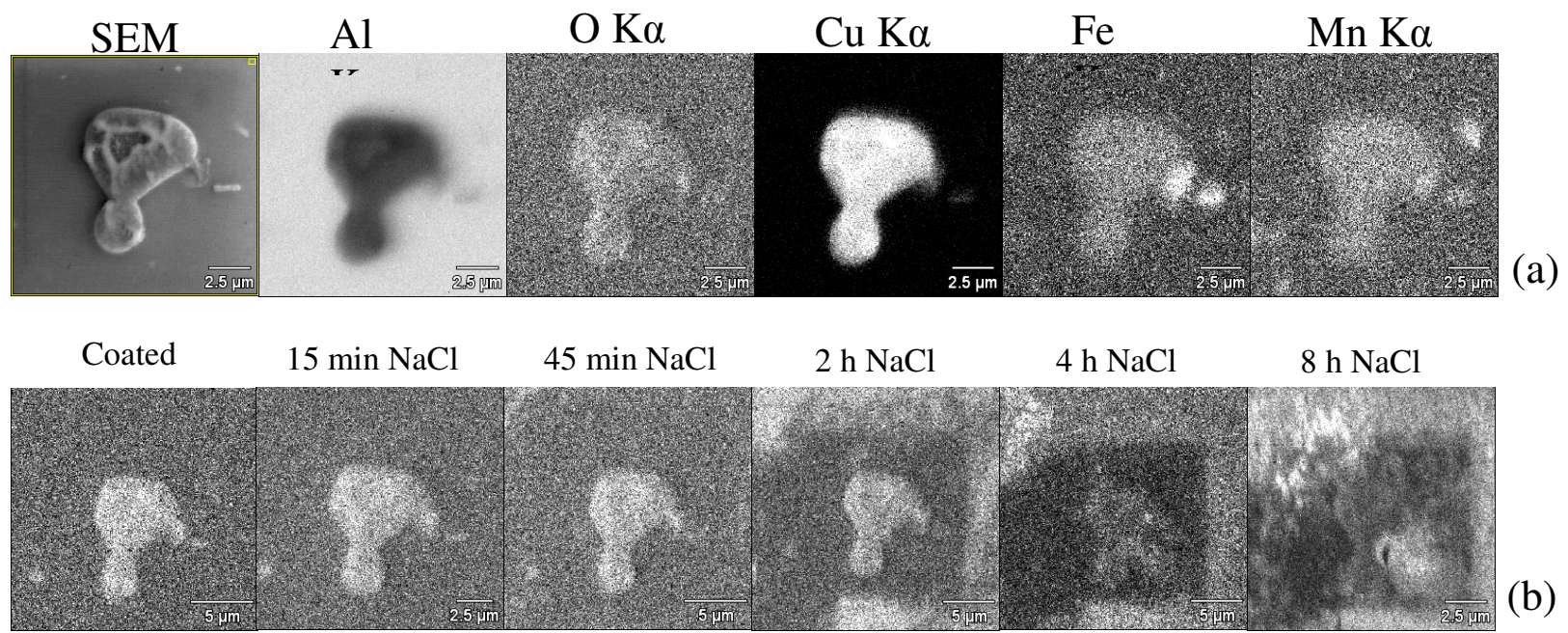

(b)

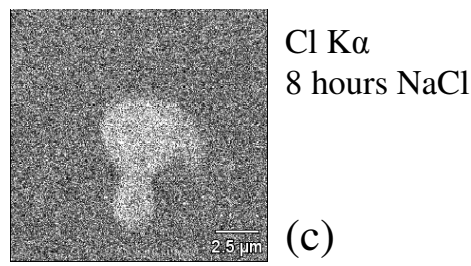

Figura 4: (a) SEM micrograph and EDX maps of Al, O, Cu, Fe and Mn from the just polished surface; (b) EDX maps sequence of oxygen at different times of immersion in the $3.5 \% \mathrm{NaCl}$ solution; (c) $\mathrm{Cl} \mathrm{K \alpha}$ map after 8 hours in the solution. 